\title{
Addition of transcatheter arterial chemoembolization decreased local recurrence but had no survival benefit to percutaneous ethanol injection therapy for patients with small hepatocellular carcinoma: A multicenter randomized control study
}

\author{
AKIRA MIZUKI $^{1}$, MASAYUKI TATEMICHI $^{1,2}$, NOBUHIRO TSUKADA $^{1}$, RYOUSUKE NAGAMATSU $^{4}$, \\ MITSUHIKO KAWAGUCHI ${ }^{5}$, TATSUYA ITOSHIMA ${ }^{5}$, SHIGEKI MARUYAMA $^{6}$, ATSUHIKO SATOU ${ }^{7}$, \\ YASUHISA IMARI $^{8}$, TOSHIHARU KAWATOKO ${ }^{9}$, JUNYA SHIMONO ${ }^{9}$ and HIROSHI NAGATA ${ }^{3}$ \\ SAISEIKAI ASSOCIATION FOR THE STUDY OF LIVER DISEASES (SASLD)
}

\begin{abstract}
${ }^{1}$ Department of Internal Medicine, Saiseikai Central Hospital; ${ }^{2}$ Department of Hygiene and Preventive Medicine, School of Medicine, Showa University; ${ }^{3}$ Department of Internal Medicine, School of Medicine, Keio University, Tokyo; ${ }^{4}$ Department of Internal Medicine, Nakatsu Saiseikai Hospital, Osaka; ${ }^{5}$ Department of Internal Medicine, Okayama Saiseikai Hospital, Okayama; ${ }^{6}$ Department of Gastroenterology, Gotsu General Hospital, Shimane

${ }^{7}$ Department of Internal Medicine, Kagawa Saiseikai Hospital, Kagawa; ${ }^{8}$ Department of Internal Medicine, Fukuoka Prefecture Yahata General Hospital; ${ }^{9}$ Department of Internal Medicine,
\end{abstract}

Fukuoka Saiseikai Hospital, Fukuoka, Japan

Received April 15, 2010; Accepted June 18, 2010

DOI: 10.3892/ol_00000151

\begin{abstract}
To assess the efficacy of the additional treatment of transcatheter arterial chemoembolization (TACE) to percutaneous ethanol injection (PEI) therapy for relatively small hepatocellular carcinomas (HCCs), a multicenter randomized control study (RCT) was performed. We conducted an RCT and follow-up study during the enrollment period from 1997 to 1999. Newly diagnosed patients with one to three HCC tumors measuring from 2 to $4 \mathrm{~cm}$ ( $4 \mathrm{~cm}$ maximum) in diameter were enrolled. A total of 30 patients initially underwent a combination TACE-PEI or PEI-alone therapies at eight randomly assigned Japanese hospitals. However, 3 patients withdrew. Of the 27 remaining patients, 13 were treated with the combination TACE-PEI therapy and 14 with PEI therapy alone. The patients were observed over several months [median (interquartile range) 33.2 (24.6) months]. There were no significant differences in the background of the patients between the two groups. Among the patients treated with TACE-PEI, the development of a local residual tumor was of significantly lower occurence, compared to the group receiving PEI alone (7.6 and $42.9 \%$, respectively; $\mathrm{P}=0.024)$. However, the mean cancer-free time (absence of local or multiple nodule recurrence) or patient
\end{abstract}

Correspondence to: Dr Akira Mizuki, Department of Internal Medicine, Saiseikai Central Hospital, Tokyo, Japan

E-mail: amizuki@highway.ne.jp

Key words: hepatocellular carcinoma, embolization, ethanol injection, combination therapy, multicenter randomized controlled study survival time was not significantly different between the two groups [PEI alone vs. TACE-PEI: cancer-free time 16.7 (95\% CI 7.3-26.0) vs. 22.9 months (95\% CI 12.4-33.4); survival time 57.2 (95\% CI 37.2-77.2) vs. 42.4 months (95\% CI 29.2-55.6)]. Although the combination of TACE and PEI had significant effects on the local tumor control, no efficacy of the addition of TACE to PEI was noted in the prognosis among patients with relatively small HCC tumors.

\section{Introduction}

In Japan, hepatocellular carcinoma (HCC) is a major health concern with an incidence of two million patients infected with hepatitis $\mathrm{C}$ virus (HCV) and with 7-8\% of patients with liver cirrhosis developing de novo HCC every year. Moreover, approximately 35,000 patients with HCC succumbed to the disease in 2009. A total of 70-80\% of HCC patients are infected with HCV and approximately $20 \%$ with hepatitis B virus (HBV) (1). It is estimated that the number of HCC patients may increase in the next 10 years. Therefore, the establishment of effective treatment modalities for HCC is imperative.

Percutaneous ethanol injection (PEI) therapy is a useful type of therapy for patients with small HCC, particularly for those with poor hepatic functional reserve $(2,3)$. PEI therapy involves the injection of absolute ethanol into HCC using ultrasound guidance, resulting in cellular dehydration, coagulation necrosis and vascular thrombosis within the treated tumor (4). Patient outcome for PEI therapy is comparable to the outcome of patients who undergo surgical resection $(5,6)$. However, the recurrence of primary HCCs after PEI is common, and the rate of local residual recurrence after PEI therapy is reported 
to range from 14 to $44 \%$ (7-10). Therefore, to control local recurrence, combination therapy with transcatheter arterial chemoembolization (TACE) and PEI has been proposed.

TACE is widely used and is considered to be an effective conservative treatment for HCCs. Embolization of the hepatic artery results in selective ischemic necrosis of the tumor tissue (11). However, complete necrosis of the tumor by embolization of the hepatic artery alone is almost impossible to achieve (12).

A number of clinical studies examined the non-surgical treatment of small HCCs including TACE alone, PEI alone and combined therapy with TACE and PEI (13-18). Certain investigators reported the superior efficacy of combined TACE-PEI therapy, compared to PEI alone. Koda et al attempted to clarify the efficacy of combination TACE-PEI therapy in patients with small HCCs $(<3 \mathrm{~cm})$ using randomized assignment (19). The results, however, revealed that patient survival was not different between combined TACE-PEI therapy and PEI therapy alone. Stratified analysis showed that for patients bearing HCC tumors $<2 \mathrm{~cm}$, combined TACE-PEI therapy was superior to PEI alone. Consequently, the efficacy of additional TACE to PEI as a recommended treatment for $\mathrm{HCCs}>2 \mathrm{~cm}$ has yet to be determined.

Thus, using multicenter randomized assignment, this study was conducted to examine the efficacy of TACE-PEI therapy instead of PEI alone for patients with relatively small HCC tumors, $2-4 \mathrm{~cm}$ in diameter.

\section{Patients and methods}

Study design. This was a multicenter randomized control (RCT) study. The study protocol was approved by the review board of each hospital, and all patients provided informed written consent.

Patients. Between July 1997 and April 1999, patients diagnosed with small HCCs for the first time were eligible to be enrolled as study subjects. The criteria for enrollment to this study were: i) age <70 years; ii) HCC nodules measuring $2-4 \mathrm{~cm}$ in maximum diameter; iii) number of $\mathrm{HCC}$ nodules $\leq 3$; iv) no portal thrombosis or extrahepatic metastasis; v) hypervascular nodules, as determined by dynamic computed tomography (CT) scan and/or arteriography; and vi) no previous treatment for HCC prior to entry. Exclusion criteria included any severe comorbidity (such as uncontrolled diabetes mellitus, heart failure, renal failure or other cancer), as well as any patient who was unable to understand the protocol or manage self-care.

The diagnosis of HCC was made by dynamic CT and/ or abdominal sonography. To assist the diagnosis of HCC, a needle biopsy was performed in all 27 patients. Tumor vascularity was also evaluated by dynamic CT and/or angiography from the hepatic artery.

Randomization was performed using a sealed-envelope method. Patients were divided into two groups: the TACE-PEI group, in which patients were treated with TACE followed by PEI and the PEI-alone group, in which patients were treated with PEI therapy alone.

Treatment procedure. Patients with HCC were treated by trained specialists at each institution. The precise techniques of ethanol injection are described elsewhere (7). Briefly, after local anesthesia, one 21-gauge needle was inserted into the lesion under ultrasound (US) guidance, and absolute ethanol was injected. In one session, 2-8 $\mathrm{ml}$ of ethanol was injected into several sites in and around the lesion according to the lesion size. After the procedure, the patients remained in bed for $3 \mathrm{~h}$. This procedure was performed twice a week. The treatment was repeated until dynamic CT demonstrated entire tumor necrosis.

In addition, TACE [precise techniques are described elsewhere $(12,13)]$ was performed by super-selectively introducing a catheter into the hepatic artery that fed the tumor. A mixture of an ionized oil and doxorubicin hydrochloride (0.6-1.0 mg per $\mathrm{kg}$ of body weight) was injected, followed by a gelatin sponge.

Diagnosis of the remaining tumors was based on image findings, particularly dynamic CT. In addition, the positivity of serum $\alpha$-fetoprotein (AFP $>10 \mathrm{ng} / \mathrm{ml}$ ) or serum protein induced by vitamin $\mathrm{K}$ absence II (PIVKA-II $>40 \mathrm{mAU} / \mathrm{ml}$ ) facilitated the diagnosis.

Follow-up. The patients were under regular observation for the detection of recurrence by measurement of tumor markers (AFP and/or PIVKA-II), ultrasonography and/or dynamic CT scans every 3 months. The primary endpoint was a recurrence indicated in any of the above examinations. The secondary endpoint was patient death. The recurrence of HCC was classified as local residual or new nodular recurrence in lesions other than the tumor treated. Local recurrence was defined as tumors within or adjacent to the tumor being treated. The recurrent tumors were treated with PEI or TACE-PEI. In the PEI-alone group, however, TACE was performed when $\geq 3$ recurrent tumors developed.

Statistical analysis. The statistical significance of the patient characteristics between the two groups was determined by the Chi-square or Mann-Whitney U test. The mean cancer-free time and survival time were calculated using the Kaplan-Meier method, and significance was determined by the generalized Wilcoxon's test. $\mathrm{P}<0.05$ was considered to be significant.

\section{Results}

A total of 30 patients fulfilled the criteria for enrollment in this study. Patients were stratified and randomized into two treatment arms: 16 patients were treated with a combination of TACE and PEI (TACE-PEI group) and 14 received only PEI therapy (PEI-alone group). However, three patients withdrew from the study, and the final number of patients analyzed was 27 (TACE-PEI group, 13; PEI-alone group, 14). Of the 27 patients, 4 had cirrhosis and 23 had chronic hepatitis. Hepatitis B surface antigen was positive in 5 of the 27 patients (18.5\%) and the HCV antibody was positive in 20 of the 27 patients $(74.1 \%)$. No significant differences were noted between the two groups in the baseline characteristics (Table I).

The median (interquartile range) follow-up period was 33.2 (24.6) months [TACE-PEI group, 39.7 (46.7) months and PEI alone group, 33 (42.7) months].

Primary endpoint: Recurrence. Tumor recurrence was detected in 10 patients treated with PEI alone and in 11 patients 
Table I. Clinical characteristics according to the treatment group.

\begin{tabular}{|c|c|c|c|}
\hline & PEI $(n=14)$ & TACE-PEI $(n=13)$ & P-value \\
\hline Age (years) $($ mean $\pm S D)$ & $63.6 \pm 6.2$ & $65.8 \pm 7.3$ & NS \\
\hline Gender $(\mathrm{M} / \mathrm{F})$ & $7 / 7$ & $9 / 4$ & NS \\
\hline \multicolumn{4}{|l|}{ Etiology of liver disease } \\
\hline $\mathrm{HBV}$ & 3 & 0 & \\
\hline $\mathrm{HCV}$ & 9 & 9 & \\
\hline $\mathrm{HBV}+\mathrm{HCV}$ & 1 & 1 & \\
\hline NBNC & 2 & 3 & NS \\
\hline Chronic hepatitis & 13 & 10 & \\
\hline Cirrhosis & 1 & 3 & NS \\
\hline Albumin (g/dl) & $3.5 \pm 0.3$ & $3.8 \pm 0.4$ & NS \\
\hline Total bilirubin (mg/dl) & $1.1 \pm 0.6$ & $1.2 \pm 0.8$ & NS \\
\hline ALT (UI/l) & $82 \pm 50$ & $145 \pm 99$ & NS \\
\hline AST (UI/l) & $65 \pm 53$ & $129 \pm 65$ & NS \\
\hline Prothrombin time $(\%)$ & $63 \pm 3.6$ & $77 \pm 15$ & NS \\
\hline$\alpha$-fetoprotein (ng/ml) [median (range)] & $13(4-97)$ & $16(4-373)$ & NS \\
\hline \multicolumn{4}{|l|}{ Tumor lesions } \\
\hline Single nodule & 11 & 8 & \\
\hline 2-3 nodules & 3 & 5 & NS \\
\hline Greatest tumor dimension (mm) & $26.4 \pm 7.4$ & $26.5 \pm 6.8$ & NS \\
\hline
\end{tabular}

TACE, transcatheter arterial chemoembolization; PEI, percutaneous ethanol injection; M, male; F, female; NS, not significant; HBV, hepatitis B virus; $\mathrm{HCV}$, hepatitis $\mathrm{C}$ virus; $\mathrm{NBNC}$, non $\mathrm{HBV}$-non $\mathrm{HCV}$; ALT, alanine aminotransferase and AST, aspartate aminotransferase.

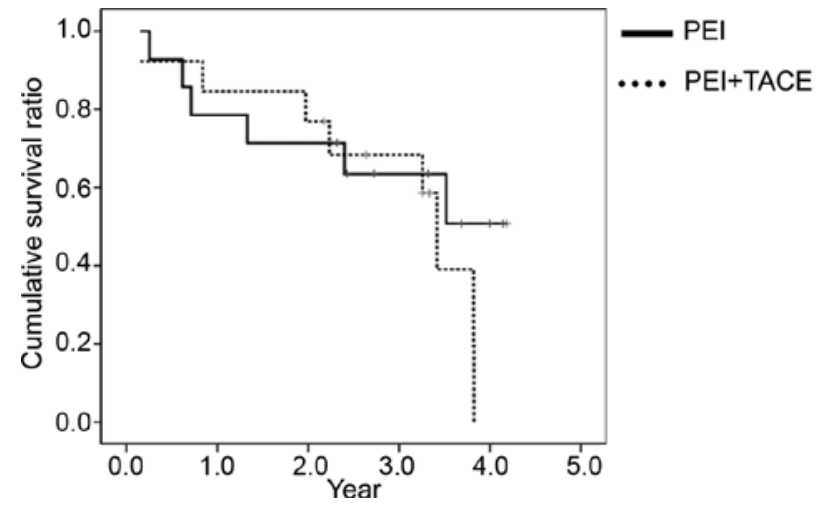

Figure 1. Comparison of the cumulative cancer-free time between the group that received TACE-PEI and the group that received PEI therapy alone. The mean cancer-free time was 16.7 months (95\% CI 7.3-26.0) for the PEI-alone group and 22.9 months (95\% CI 12.4-33.4) for the TACE-PEI group. No significant difference was noted between the two groups.

treated with TACE-PEI. The cumulative cancer-free time was calculated using the Kaplan-Meier method (Fig. 1). The mean cancer-free time was 16.7 months (95\% CI 7.3-26.0) for the PEI alone group and 22.9 months (95\% CI 12.4-33.4) for the TACE-PEI group. No significant difference was found between the two groups. However, the pattern of recurrence was significantly different $(\mathrm{P}<0.05)$. During follow-up, the detection of a local residual lesion was observed in 1 of 13 nodules $(7.6 \%)$ in the TACE-PEI group and in 6 of 13 nodules $(46.1 \%)$

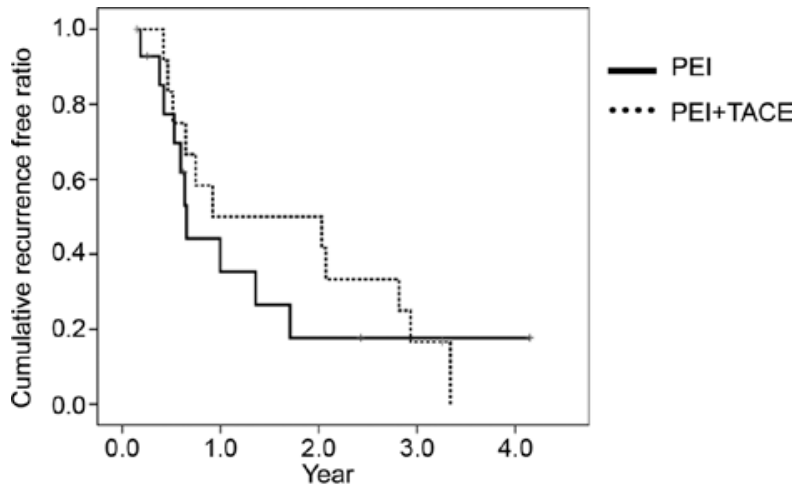

Figure 2. Comparison of the survival rates between the TACE-PEI group and the PEI-alone group. The mean survival time of the TACE-PEI group was 42.4 months (95\% CI 29.2-55.6) and that of the PEI-alone group was 57.2 months (95\% CI 37.2-77.2). No significant difference was found between the two groups.

in the PEI-alone group. No local residual tumor was detected after 2 years of follow-up in the TACE-PEI group. On the other hand, new nodular recurrences were observed in 8 of 13 patients $(61.5 \%)$ in the TACE-PEI group and in 3 of 14 patients $(21.4 \%)$ in the PEI-alone group.

Secondary endpoint: Death. Of the 13 patients $(61.5 \%)$, 8 treated with TACE-PEI and 6 of the 14 patients $(44 \%)$ treated with PEI alone succumbed to the disease during the 
follow-up period. In the TACE-PEI group, causes of death included development of HCC in 2 patients, variceral bleeding in 3 patients and hepatic failure in 3 patients. In the PEI-alone group, causes of death were development of $\mathrm{HCC}$ in 2 patients, hepatic failure in 3 patients and other diseases (tuberculosis) in 1 patient. The cumulative survival curves of the two groups are shown in Fig. 2. The mean patient survival time of the TACE-PEI group was 42.4 months (95\% CI 29.2-55.6) and that of the PEI-alone group was 57.2 months (95\% CI 37.2-77.2). No significant difference was noted between the two groups.

Adverse events. In all 30 cases, serious adverse effects or complications, such as acute liver failure, liver infarction, abscess, cholecystitis, gastrointestinal mucosal lesions, pulmonary embolism, variceral bleeding, iatrogenic dissection or perforation of the celiac artery and its branches, were not related to treatment with TACE and/or PEI.

\section{Discussion}

This RCT study failed to show the anticipated efficacy of TACE-PET therapy compared to PEI treatment alone on survival time for patients with relatively small HCCs of 2-4 cm in diameter. Together with the previous RCT result by Koda $e t a l$, it was found that a tumor size smaller than 2 $\mathrm{cm}$ may be critical in obtaining significant effectiveness by combining TACE therapy to PEI (19).

The present study showed marked differences in recurrence patterns after initial treatment. Our results indicate that TACE-PEI is superior to PEI therapy alone regarding local tumor control. The addition of TACE, however, evoked new tumors in different lesions other than the original tumor. We believe that the induction of growth factors such as VEGF and HGF (20-23), due to ischemia by TACE, are involved in the development of new nodules. A liver with HCCs larger than $2 \mathrm{~cm}$ in diameter may be prone to develop HCCs in whole liver lesions. Stimulation by TACE may enhance the progression of small nodules that are not detected by $\mathrm{CT}$ examination. When the stage of HCC is evaluated using more sensitive methods, such as CT during arterial portography and/or superparamagnetic iron oxide-enhanced gradient-recalled echo MRI (24-29), extremely small focal nodules can be detected.

The main causes of patient death in the present study were related to hepatic failure and not to tumor progression in either group. Although it is reported that TACE improves tumor control in large-size HCCs, our data suggest that the prognosis of patients with $\mathrm{HCCs}$ of $2-4 \mathrm{~cm}$ in diameter depends on residual liver function, and not on tumor progression. No statistical significance was found in the present study which showed that the mean patient survival time was shorter in the TACE-PEI group than that in the PEI-alone group. Therefore, local tumor control may not directly contribute to patient survival time.

A number of limitations should be noted. Although patients were enrolled at different sites, a relatively small number of patients was unable to participate, and the follow-up period was short. All but two tumors were virus-related HCCs. Recently, the incidence of HCC from non-alcoholic steatohepatitis has been on the increase and its characteristics are reportedly different from HCCs resulting from $\mathrm{HCV}$ and $\mathrm{HBV}$
(30). However, this study used random assignment, providing us with important information regarding the treatment of relatively small-size virus-related HCCs. For HCCs of 2-4 $\mathrm{cm}$ in diameter, the additional TACE to PEI did not markedly improve patient survival. Moreover, the additional TACE treatment appeared to shorten the patient survival time as the treatment did not (at least notably) damage residual liver function and stimulated new tumor growth in lesions other than the primary one. Additionally, other modalities, such as radio frequency ablation (RFA), are available. Such treatment modalities are considered to be superior to PEI in local tumor control and attack tumors in a pin-point manner (31-35). Thus, our data suggest that RFA alone as well as PEI may be recommended in the treatment of relatively small $\mathrm{HCCs}$ of $2-4 \mathrm{~cm}$ in diameter.

\section{Acknowledgements}

We would like to thank Dr Hidetsugu Saitou who supervised the study.

\section{References}

1. Okita K: Clinical aspects of hepatocellular carcinoma in Japan. Intern Med 45: 229-233, 2006.

2. Livraghi T, Festi D, Monti F, Salmi A and Vettori C: US-guided percutaneous alcohol injection of small hepatic and abdominal tumors. Radiology 161: 309-312, 1986.

3. Ohoto M, Ebara M, Watanabe Y, et al: Percutaneous ethanol injection (PEI) therapy for small hepatocellular carcinoma. Evaluation of its utility on the basis of tumor-images and survival after therapy. Jpn J Med Imaging 7: 25-33, 1988.

4. Livraghi T, Giorgio A, Marin G, et al: Hepatocellular carcinoma and cirrhosis in 746 patients: long-term results of percutaneous ethanol injection. Radiology 197: 101-108, 1995.

5. Kotoh K, Sakai H, Sakamoto S, Nakayama S, Satoh M, Morotomi I and Nawata $\mathrm{H}$ : The effect of percutaneous ethanol injection therapy on small solitary hepatocellular carcinoma is comparable to that of hepatectomy. Am J Gastroenterol 89: 194-198, 1994.

6. Castells A, Bruix J, Bru C, et al: Treatment of small hepatocellular carcinoma in cirrhotic patients: a cohort study comparing surgical resection and percutaneous ethanol injection. Hepatology 18: 1121-1126, 1993.

7. Koda M, Murawaki Y, Mitsuda A, et al: Predictive factors for intrahepatic recurrence after percutaneous ethanol injection therapy for small hepatocellular carcinoma. Cancer 88: 529-537, 2000.

8. Ishii H, Okada S, Nose H, et al: Local recurrence of hepatocellular carcinoma after percutaneous ethanol injection. Cancer 77: 1792-1796, 1996.

9. Castellano L, Calandra M, del Vecchio Blanco C and de Sio I: Predictive factors of survival and intrahepatic recurrence of hepatocellular carcinoma in cirrhosis after percutaneous ethanol injection: analysis of 71 patients. J Hepatol 27: 862-870, 1997.

10. Ohnishi K, Yoshioka H, Ito S and Fujiwara K: Prospective randomized controlled trial comparing percutaneous acetic acid injection and percutaneous ethanol injection for small hepatocellular carcinoma. Hepatology 27: 67-72, 1998.

11. Doyon D, Mouzon A, Jourde AM, Regensberg C and Frileux C: [Hepatic, arterial embolization in patients with malignant liver tumours]. Ann Radiol 17: 593-603, 1974.

12. Yamada R, Sato M, Kawabata M, Nakatsuka H, Nakamura $K$ and Takashima S: Hepatic artery embolization in 120 patients with unresectable hepatoma. Radiology 148: 397-401, 1983.

13. Choi BI, Kim HC, Han JK, et al: Therapeutic effect of transcatheter oily chemoembolization therapy for encapsulated nodular hepatocellular carcinoma: CT and pathologic findings. Radiology 182: 709-713, 1992.

14. Bruix J, Llovet JM, Castells A, et al: Transarterial embolization versus symptomatic treatment in patients with advanced hepatocellular carcinoma: results of a randomized, controlled trial in a single institution. Hepatology 27: 1578-1583, 1998. 
15. Kobayashi S, Nakanuma Y, Terada T and Matsui O: Postmortem survey of bile duct necrosis and biloma in hepatocellular carcinoma after transcatheter arterial chemoembolization therapy: relevance to microvascular damage of peribiliary capillary plexus. Am J Gastroenterol 88: 1410-1415, 1993.

16. Chung JW, Park JH, Han JK, Choi BI, Han MC, Lee HS and Kim CY: Hepatic tumors: predisposing factors for complications of transcatheter oily chemoembolization. Radiology 198: 33-40, 1996.

17. Sakamoto I, Aso N, Nagaoka K, et al: Complications associated with transcatheter arterial embolization for hepatic tumors. Radiographics 18: 605-619, 1998.

18. Tanaka K, Okazaki H, Nakamura S, et al: Hepatocellular carcinoma: treatment with a combination therapy of transcatheter arterial embolization and percutaneous ethanol injection. Radiology 179: 713-717, 1991.

19. Koda M, Murawaki Y, Mitsuda A, et al: Combination therapy with transcatheter arterial chemoembolization and percutaneous ethanol injection compared with percutaneous ethanol injection alone for patients with small hepatocellular carcinoma: a randomized control study. Cancer 92: 1516-1524, 2001.

20. Li X, Feng GS, Zheng CS, Zhuo CK and Liu X: Expression of plasma vascular endotherial growth factor in patients with hepatocellular carcinoma and effect of transcatheter arterial chemoembolization therapy of plasma vascular endothelial growth factor level. World J Gastroenterol 10: 2878-2882, 2004.

21. Suzuki H, Mori M, Kawaguchi C, Adachi M, Miura S and Ishii $\mathrm{H}$ : Serum vascular endothelial growth factor in the course of transcatheter arterial embolization of hepatocellular carcinoma. Int J Oncol 14: 1087-1090, 1999.

22. Kobayashi N, Ishii M, Ueno Y, Kisara N, Chida N, Iwasaki T and Toyota T: Coexpression of Bcl-2 protein and vascular endothelial growth factor in hepatocellular carcinomas treated by chemoembolization. Liver 19: 25-31, 1999.

23. Gupta S, Kobayashi S, Phongkitkarun S, Broemeling LD and Kan Z: Effect of transcatheter hepatic arterial embolization on angiogenesis in an animal model. Invest Radiol 4: 516-521, 2006.

24. Kim HC, Kim TK, Sung KB, et al: CT during hepatic arteriography and portography: an illustrative review. Radiographics 22 : 1041-1051, 2002.

25. Heiken JP, Weyman PJ, Lee JK, Balfe DM, Picus D, Brunt EM and Flye MW: Detection of focal hepatic masses: prospective evaluation with CT, delayed CT, CT during arterial portography, and MR imaging. Radiology 171: 47-51, 1989.
26. Yu JS, Kim KW, Lee JT and Yoo HS: MR imaging during arterial portography for assessment of hepatocellular carcinoma: comparison with CT during arterial portography. Am J Roentgenol 170: 1501-1506, 1998

27. Kanematsu M, Hoshi $\mathrm{H}$ and Murakami T: Detection of hepatocellular carcinoma in patients with cirrhosis: MR imaging versus angiographically assisted helical CT. Am J Roentgenol 169: $1507-1515,1997$.

28. Murakami T, Oi H, Hori M, et al: Helical CT during arterial portography and hepatic arteriography for detecting hypervascular hepatocellular carcinoma. Am J Roentgenol 169: 131-135, 1997.

29. Kanematsu M, Hoshi H, Imaeda T, Murakami T, Inaba $Y$, Yokoyama $\mathrm{R}$ and Nakamura $\mathrm{H}$ : Detection and characterization of hepatic tumors: value of combined helical CT hepatic arteriography and CT during arterial portography. Am J Roentgenol 168: 1193-1198, 1997.

30. Page JM and Harrison SA: NASH and HCC. Clin Liver Dis 13: 631-647, 2009.

31. Shiina S, Teratani T, Obi S, et al: A randomized controlled trial of radiofrequency ablation with ethanol injection for small hepatocellular carcinoma. Gastroenterology 129: 122-130, 2005.

32. Lin SM, Lin CJ, Lin CC, Hsu CW and Chen YC: Radiofrequency ablation improves prognosis compared with ethanol injection for hepatocellular carcinoma $\leq 4 \mathrm{~cm}$. Gastroenterology 127: 1714-1723, 2004

33. Lencioni RA, Allgaier HP, Cioni D, et al: Small hepatocellular carcinoma in cirrhosis: randomized comparison of radio-frequency thermal ablation versus percutaneous ethanol injection. Radiology 228: 235-240, 2003.

34. Livraghi T, Goldberg SN, Lazzaroni S, Meloni F, Solbiati L and Gazelle GS: Small hepatocellular carcinoma: treatment with radio-frequency ablation versus ethanol injection. Radiology 210: 655-661, 1999.

35. Brunello F, Veltri A, Carucci P, et al: Radiofrequency ablation versus ethanol injection for early hepatocellular carcinoma: a randomized controlled trial. Scand J Gastroenterol 43: 727-735, 2008. 